STRUCTURAL BIOLOGY

ISSN 2059-7983

Received 27 October 2021

Accepted 12 January 2022

Edited by B. Kobe, University of Queensland, Australia

Keywords: InlB; Listeria monocytogenes; binding sites; crystal contacts; crystallization propensity; protein-protein interactions.

PDB references: $\ln \mid B_{392}$, wild type, $7 p v 9$; InlB $392-T 332 \mathrm{E}$, crystal form I, 7pv8; crystal form II, $7 \mathrm{nms}$

Supporting information: this article has supporting information at journals.iucr.org/d

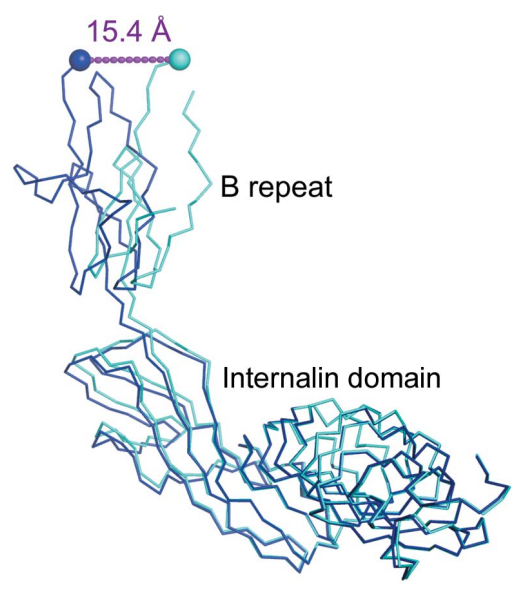

OPEN $\odot$ ACCESS

\section{A recurring packing contact in crystals of InlB pinpoints functional binding sites in the internalin domain and the $B$ repeat}

\author{
Christina Geerds, Willem M. Bleymüller, Timo Meyer, Christiane Widmann and \\ Hartmut H. Niemann*
}

Department of Chemistry, Bielefeld University, Universitätsstrasse 25, 33615 Bielefeld, Germany. *Correspondence e-mail: hartmut.niemann@uni-bielefeld.decrystal form II, 7nms

InlB, a bacterial agonist of the human receptor tyrosine kinase MET, consists of an N-terminal internalin domain, a central B repeat and three C-terminal GW domains. In all previous structures of full-length InlB or an InlB construct lacking the $\mathrm{GW}$ domains $\left(\mathrm{InlB}_{392}\right)$, there was no interpretable electron density for the B repeat. Here, three $\mathrm{InlB}_{392}$ crystal structures in which the B repeat is resolved are described. These are the first structures to reveal the relative orientation of the internalin domain and the B repeat. A wild-type structure and two structures of the T332E variant together contain five crystallographically independent molecules. Surprisingly, the threonine-to-glutamate substitution in the B repeat substantially improved the crystallization propensity and crystal quality of the T332E variant. The internalin domain and B repeat are quite rigid internally, but are flexibly linked to each other. The new structures show that inter-domain flexibility is the most likely cause of the missing electron density for the B repeat in previous InlB structures. A potential binding groove between B-repeat strand $\beta 2$ and an adjacent loop forms an important crystal contact in all five crystallographically independent chains. This region may represent a hydrophobic 'sticky patch' that supports protein-protein interactions. This assumption agrees with the previous finding that all known inactivating point mutations in the $\mathrm{B}$ repeat lie within strand $\beta 2$. The groove formed by strand $\beta 2$ and the adjacent loop may thus represent a functionally important proteinprotein interaction site in the $\mathrm{B}$ repeat.

\section{Introduction}

InlB is an invasion protein that is located on the surface of the bacterium Listeria monocytogenes (Dramsi et al., 1995; Lingnau et al., 1995). As a facultative intracellular pathogen, L. monocytogenes can stimulate its own uptake by nonphagocytic cells such as epithelial or endothelial cells (PizarroCerdá et al., 2012). Binding of InlB to the receptor tyrosine kinase MET on host cells is one way to induce internalization of bacteria (Shen et al., 2000). MET acts as a receptor for hepatocyte growth factor/scatter factor $(\mathrm{HGF} / \mathrm{SF})$ and is essential during mammalian development (Birchmeier et al., 2003). MET also contributes to tissue regeneration and wound healing, while MET deregulation can promote cancer metastasis (Trusolino et al., 2010). Cellular phenotypes in response to InlB stimulation resemble those observed upon MET activation by HGF/SF. They include cell motility (for example cell scatter of clonally growing MDCK or HT-29 cells) and cell proliferation (Shen et al., 2000; Niemann et al., 2007). At the molecular level, InlB induces MET phosphorylation and the 
activation of downstream signalling pathways such as phosphorylation of extracellular signal-regulated kinase (ERK) or activation of phosphoinositide 3-kinase (PI3K) and phosphorylation of protein kinase B (PKB)/AKT (Copp et al., 2003; Seveau et al., 2007).

Mature InlB is a 595-amino-acid, multi-domain protein (Fig. 1a). The N-terminal internalin domain (amino acids 36321; $\mathrm{InlB}_{321}$ ) is characterized by a central leucine-rich repeat (LRR) region that binds to the first Ig-like IPT domain of MET with high affinity (Niemann et al., 2007). The LRR region is flanked $\mathrm{N}$-terminally by a helical cap and C-terminally by an Ig-like inter-repeat (IR) region (Schubert et al., 2001). The

\begin{tabular}{|lll|l|l|l|l|}
36 & 85 & 239 & 321 & 392 & 468 & 551 \\
\hline Cap & LRR & IR & B & GW1 & GW2 & GW3 \\
\hline \multicolumn{7}{|c|}{ Internalin domain }
\end{tabular}

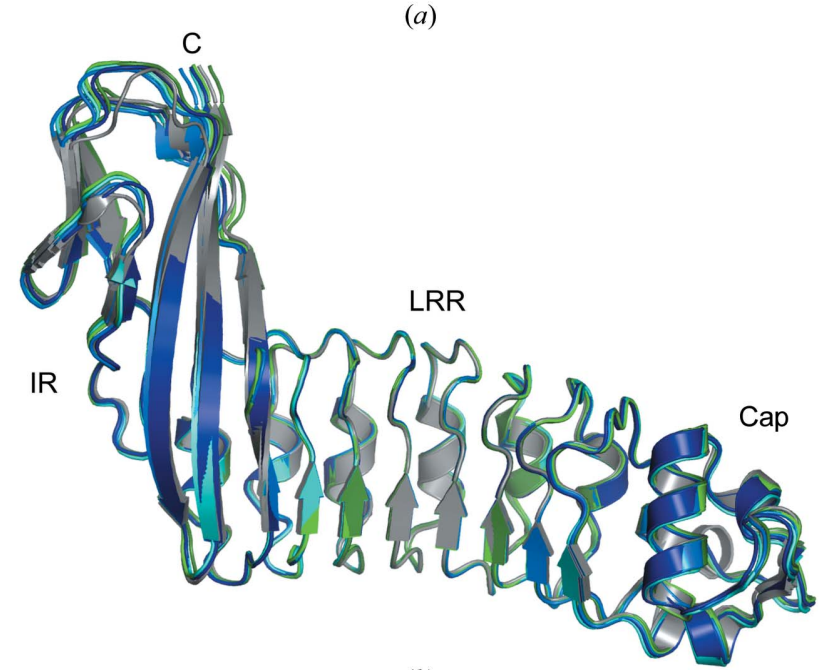

(b)

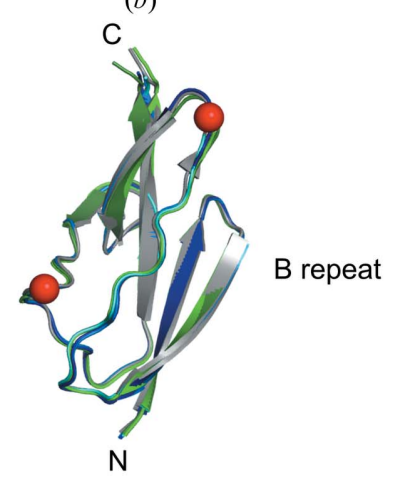

(c)

Figure 1

Domain structure and overlay of individual domains with previous highresolution structures. (a) Domain structure of InlB. The amino-acid numbers shown above indicate domain boundaries. The internalin domain consists of three structural regions: cap, LRR and IR. $(b, c)$ Chains $A, B$ and $C$ of $\mathrm{InlB}_{392}$ wt are shown in dark blue, blue and cyan, respectively. Crystal forms I and II of InlB $_{392}$ T332E are shown in green and dark green, respectively. Reference structures are shown in grey. (b) The internalin domains of all $\mathrm{InlB}_{392}$ structures were overlaid on PDB entry 1 h6t. The overlay was performed for the LRR region. (c) The B repeat of all $\mathrm{InlB}_{392}$ structures was overlaid on chain $A$ of PDB entry $2 \mathrm{y} 5 \mathrm{p}$. The red spheres indicate the $\mathrm{C}^{\alpha}$ atoms of the residues before (residue 353) and after (residue 373) the region that is not resolved in chain $C$ of $\mathrm{InlB}_{392}$ wt.
C-terminal part (amino acids 393-630) consists of three GW domains named after a conserved Gly-Trp motif. The GW domains have an SH3-like fold and a high isoelectric point (pI) of about 10 (Marino et al., 2002). The GW domains bind polyanions, including lipoteichoic acid (LTA), on the bacterial surface (Jonquières et al., 1999) and heparan sulfate of host cell surface heparan sulfate proteoglycans (HSPGs; Jonquières et al., 2001). Binding to LTA or HSPGs is mutually exclusive (Jonquières et al., 2001). InlB apparently acts as a soluble protein that induces bacterial uptake into host cells rather than as an adhesin that supports the attachment of L. monocytogenes to host cells (Jonquières et al., 2001; Banerjee et al., 2004). Binding of the GW domains to host HSPGs enhances MET activation through the internalin domain (Banerjee et al., 2004), presumably via an avidity effect or receptor clustering (Jonquières et al., 2001; Niemann et al., 2007). On their own or provided in trans with $\mathrm{InlB}_{392}$, the GW domains showed no effect in cellular assays (Banerjee et al., 2004).

InlB activates MET by dimerization of the MET ectodomain (Niemann, 2013). InlB-induced MET dimerization on cells was shown by fluorescence microscopy (Dietz et al., 2013; Harwardt et al., 2017; Koschut et al., 2016). The crystal structure of a 2:2 complex formed by the InlB internalin domain and a large portion of the MET extracellular domain revealed an arrangement in which two InlB molecules form a twofoldsymmetric homodimer via the convex distal surface of their LRR regions (Niemann et al., 2007; Ferraris et al., 2010). Two MET molecules sit on the outside of this InlB dimer, with each being bound to the concave 'front side' of the LRR region. Except for a small contact of two IPT2 domains, MET does not contribute to dimerization in this structure. The same 2:2 arrangement of the InlB internalin domain and MET has recently been observed in another structure obtained in the presence of a MET-binding DARPin, lending further support to this activation model (Andres et al., 2019).

InlB structures have been published, in the following order, of the LRR fragment ( $\operatorname{InlB}_{248}$; Marino et al., 1999), the internalin domain ( $\mathrm{InlB}_{321}$; Schubert et al., 2001) and the full-length protein (Marino et al., 2002). However, the B repeat was not resolved in the structure of full-length InlB. There was some electron density between the internalin and GW domains, but this could not be modelled. Therefore, we determined the structure of a fragment consisting of the internalin domain and the $\mathrm{B}$ repeat but lacking the $\mathrm{GW}$ domains $\left(\mathrm{InlB}_{392}\right.$; Ebbes $e t$ al., 2011). Again, the $B$ repeat was not resolved and this time there was no electron density at all for the B repeat, suggesting it to be highly flexible. In this crystal form, all packing contacts are formed by the internalin domain. We assume that the B repeat makes no crystal contacts at all and that it is free to move in large solvent channels. Therefore, we crystallized the B repeat alone (Ebbes et al., 2011). These crystals diffracted to high resolution $(1.3 \AA$ ) and revealed a well defined structure. The $\mathrm{B}$ repeat folds into a compact domain resembling a $\beta$-grasp fold. The only difference from a canonical $\beta$-grasp fold is the absence of the helix connecting strands $\beta 2$ and $\beta 3$. Instead, the two parallel edge $\beta$-strands are connected by an extended loop structure. While structures of all domains of 
InlB are now available, the relative orientation of the B repeat and the internalin domain remains elusive.

The B repeat is arguably the least understood domain in InlB. Several internalins contain up to four domains that are homologous to the $\mathrm{B}$ repeat, yet none of them has been functionally characterized (Bierne et al., 2007). Initial experiments with recombinant domain-deletion constructs of InlB showed that the B repeat contributes to ERK activation, but found no effect on MET phosphorylation (Copp et al., 2003). This led to the suggestion that the B repeat could bind a co-receptor and activate a second signalling pathway parallel to MET. A more detailed study revealed that $\mathrm{InlB}_{392}$ has a slightly (about twofold) higher potency in inducing MET phosphorylation than $\mathrm{InlB}_{321}$ (Banerjee et al., 2004). Thus, the $\mathrm{B}$ repeat apparently does contribute to MET activation. Cellmotility assays with canine MDCK and human cells revealed a profound effect of the B repeat. While $\mathrm{InlB}_{392}$ induced MDCK colony scatter at $10 \mathrm{n} M$ (Ebbes et al., 2011), $\mathrm{InlB}_{321}$ was unable to do so even at $1000 \mathrm{n} M$ (Ferraris et al., 2010). A construct consisting only of the B repeat and GW1-3 had no effect (Ebbes et al., 2011). In human HT-29 cells, InlB 392 was about tenfold more active than $\operatorname{InlB}_{321}$ (Bleymüller et al., 2016). In summary, the B repeat on its own shows no effect on cells. When fused to the internalin domain it enhances MET phosphorylation. Its presence increases potency even more in assays of ERK or AKT phosphorylation or cellular phenotypes such as cell motility. So far, however, all of our efforts to identify the postulated co-receptor for the B repeat have failed (Bleymüller et al., 2016).

Analysis of $\mathrm{InlB}_{392}$ variants with single or multiple substitutions on the surface of the B repeat in cellular receptoractivation assays revealed only two functionally important amino acids, both of which are located in the edge strand $\beta 2$ (Bleymüller et al., 2016). The mutation of Thr332 or Ile334 strongly impaired or completely abolished the activity of $\mathrm{InlB}_{392}$ in cellular assays. The resulting protein variants were at least 100 -fold less active than wild-type $\operatorname{InlB}_{392}\left(\operatorname{InlB}_{392}\right.$ wt). Interestingly, the $\mathrm{InlB}_{392}$ variants $\mathrm{T} 332 \mathrm{E}$ and $\mathrm{I} 334 \mathrm{D} / \mathrm{T} 336 \mathrm{~L}$ (variant $\mathrm{D}$ in Bleymüller et al., 2016) were inactive in a cellmotility assay with human HT-29 cells even at the highest concentration tested $(1000 \mathrm{n} M)$, while the internalin domain alone stimulated cell scatter at $10 \mathrm{n} M$. Thus, InlB $_{392}$ variants with a single substitution in the B repeat are substantially less active than a construct that completely lacks the $\mathrm{B}$ repeat. One possible mechanistic explanation for this perplexing result is that the wild-type B repeat contributes to MET dimerization, whereas the T332E or I334D mutation hinders dimerization of the internalin domains (Bleymüller et al., 2016). To scrutinize this hypothesis, it would be highly interesting to resolve the relative orientation of the internalin domain and to identify protein-protein interaction sites in the $\mathrm{B}$ repeat.

Here, we describe the crystallization and structure determination of wild-type $\operatorname{InlB}_{392}$ ( $\left.\mathrm{InlB}_{392 \_} \mathrm{wt}\right)$ and the inactive variant $\mathrm{InlB}_{392}$ T332E. While the wild-type protein was difficult to crystallize and the rare crystals generally showed no or rather low-resolution diffraction, we obtained crystals of $\mathrm{InlB}_{392}$ T332E under several conditions and were able to determine two structures at resolutions of 2.05 and $1.8 \AA$. Both domains are resolved in all three structures, revealing the arrangement of the internalin domain and the B repeat in InlB for the first time.

\section{Materials and methods}

\subsection{Protein crystallization}

All three proteins were expressed and purified as described in Bleymüller et al. (2016). For crystallization, the protein buffer phosphate-buffered saline (PBS), in which the proteins were stored at $-80^{\circ} \mathrm{C}$, was exchanged for crystallization buffer (10 $\mathrm{m} M$ Tris $\mathrm{pH} 8.0,20 \mathrm{mM} \mathrm{NaCl})$. All proteins were crystallized in MRC 2-well plates with drops consisting of $100 \mathrm{nl}$ protein $\left(10 \mathrm{mg} \mathrm{ml}^{-1}\right)$ and $100 \mathrm{nl}$ reservoir solution set up using a Crystal Gryphon robot (Art Robbins Instruments). All crystals were harvested directly from commercial or homemade standard screens without further optimization. $\mathrm{InlB}_{392}$ wt crystallized at $20^{\circ} \mathrm{C}$ in the MORPHEUS screen (Gorrec, 2009) condition E6 [0.1 M (HEPES sodium salt/ MOPS acid) pH 7.5, 20\%(v/v) ethylene glycol, $10 \%(w / v)$ PEG $8000,30 \mathrm{~m} M$ diethylene glycol, $30 \mathrm{~m} M$ triethylene glycol, $30 \mathrm{~m} M$ tetraethylene glycol, $30 \mathrm{~m} M$ pentaethylene glycol]. For harvesting, crystals were cryoprotected in reservoir solution to which an additional $20 \%(v / v)$ ethylene glycol was added before flash-cooling the crystal in liquid nitrogen. Crystal form I of $\mathrm{InlB}_{392}$ T332E was obtained at $4^{\circ} \mathrm{C}$ in the MORPHEUS screen condition G2 [0.1 M (imidazole/MES) pH 6.5, $20 \%(v / v)$ ethylene glycol, 10\%(w/v) PEG 8000, $20 \mathrm{mM}$ sodium formate, $20 \mathrm{~m} M$ ammonium acetate, $20 \mathrm{mM}$ sodium citrate tribasic dehydrate, $20 \mathrm{~m} M$ sodium potassium tartrate tetrahydrate, $20 \mathrm{mM}$ sodium oxamate]. These crystals were harvested directly from the crystallization drop and flashcooled in liquid nitrogen without the addition of a cryoprotectant. Crystal form II of $\mathrm{InlB}_{392}$ T332E was obtained at $4{ }^{\circ} \mathrm{C}$ in condition D3 of a homemade PEG smear screen with lowand broad-molecular-weight PEGs as described in Chaikuad et al. (2015). The reservoir consisted of $0.1 M$ succinate $\mathrm{pH} 7.0$, $0.2 \mathrm{M} \mathrm{Li}_{2} \mathrm{SO}_{4}$ and $22.5 \%$ PEG mixture consisting of equal amounts of eight low-molecular-weight PEGs (PEG 300, PEG 400, PEG 500 MME, PEG 550 MME, PEG 600, PEG 750 MME, PEG 1000 and PEG 1000 MME). These crystals were cryoprotected in reservoir solution additionally containing $15 \%$ glycerol before flash-cooling in liquid nitrogen.

\subsection{Data collection and processing}

Data for InlB $_{392}$ wt were collected on beamline P13 operated by EMBL Hamburg at the PETRA III storage ring (Cianci et al., 2017) using a PILATUS 6M detector (Dectris). For $\mathrm{InlB}_{392}$ T332E, measurements were carried out on the BL14.2 beamline at the BESSY II electron-storage ring operated by the Helmholtz-Zentrum Berlin für Materialien und Energie (Mueller et al., 2015). Data for both crystal forms of $\mathrm{InlB}_{392} \mathrm{~T}$ T322E were collected on a PILATUS $3 \mathrm{~S} 2 \mathrm{M}$ detector (Dectris). Between 240 and $360^{\circ}$ of fine-sliced data $\left(0.1^{\circ}\right.$ per frame) were collected. The data were indexed and 
Table 1

Data-collection and refinement statistics.

Values in parentheses are for the highest resolution shell.

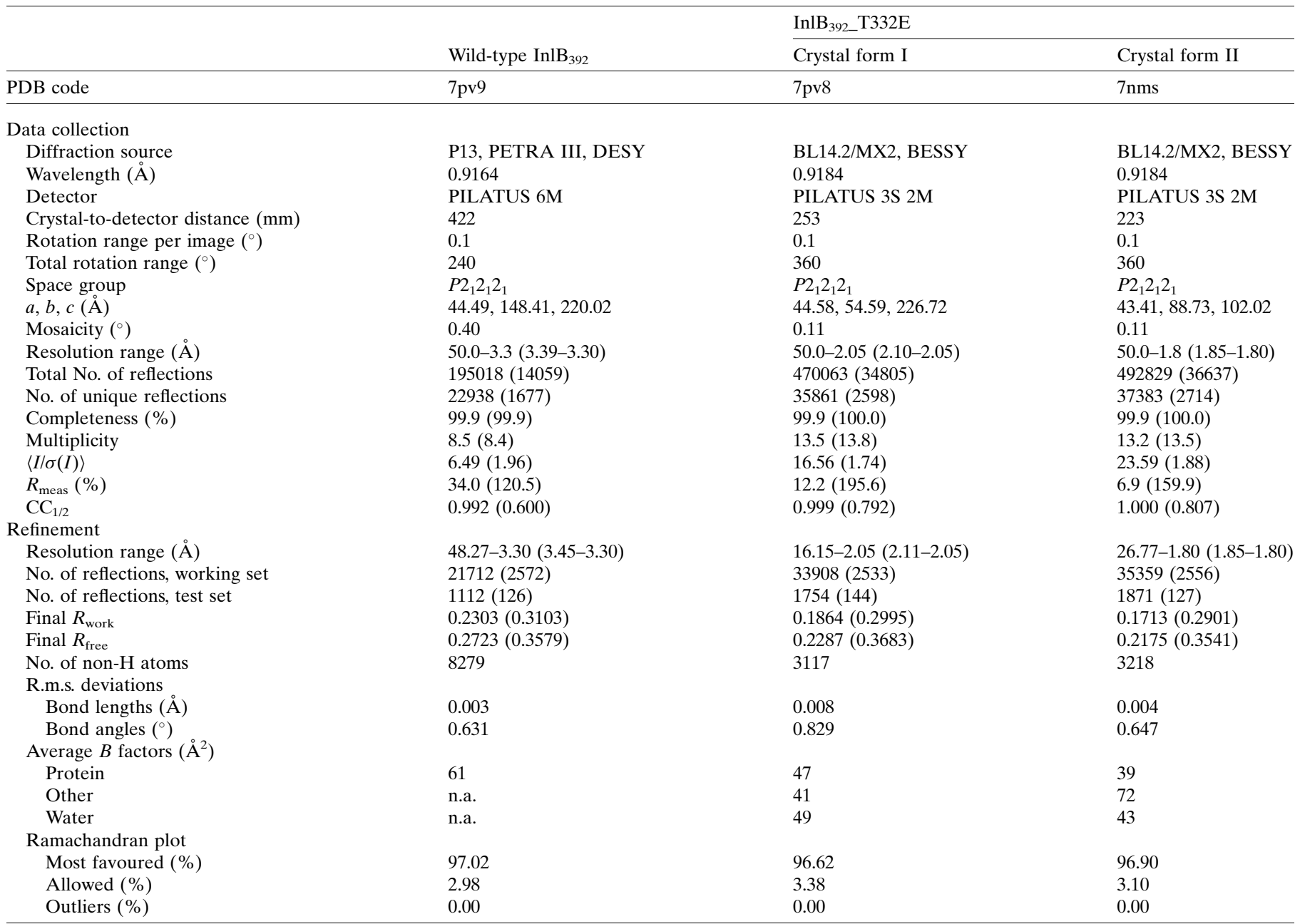

integrated with $X D S$ (Kabsch, 2010) and scaled with XSCALE using zero-dose extrapolation (Diederichs et al., 2003). Datacollection statistics are shown in Table 1.

\subsection{Structure determination and refinement}

The structures were solved by molecular replacement with Phaser (McCoy et al., 2007) from the CCP4 package (Winn et $a l ., 2011)$. The internalin domain (PDB entry 1h6t; Schubert et al., 2001) was placed first, followed by placement of the B repeat (PBD entry 2y5p; Ebbes et al., 2011). The structures were completed by iterative cycles of model building in Coot (Casañal et al., 2020) and refinement in REFMAC5 (Kovalevskiy et al., 2018) during the early stages and phenix.refine (Liebschner et al., 2019) during the final stages of rebuilding. TLS refinement was used for all three structures. For InlB $_{392}$ wt with three chains in the asymmetric unit, local noncrystallographic symmetry (NCS) restraints and restraints to InlB $_{392}$ T332E crystal form II (PDB entry $7 \mathrm{nms}$ ) as a reference model were applied. Refinement statistics are shown in Table 1.

\section{Results}

3.1. Crystallization and structure determination

We extensively screened for new crystallization conditions of wild-type InlB $_{392}$. Crystals only grew in condition E6 of a MORPHEUS screen (Gorrec, 2009) stored beyond its 'use by' date. We were unable to reproduce or optimize these crystals with homemade solutions. At $20^{\circ} \mathrm{C}$ we obtained single crystals shaped as hexagonal plates typically of about $60 \times 60 \times 10 \mu \mathrm{m}$ in size and reaching up to $100 \times 100 \times 15 \mu \mathrm{m}$. These crystals showed no diffraction even on beamlines BL14.2 of BESSY II and P13 of PETRA III. At $4^{\circ} \mathrm{C}$ we obtained thin rod-shaped crystals with a cross-section of about $10 \times 10 \mu \mathrm{m}$ and a length of $80-100 \mu \mathrm{m}$. The best crystal diffracted to $3.3 \AA$ resolution (Table 1). For flash-cooling, we had to add ethylene glycol (see Section 2) as the reservoir solution alone did not freeze clearly, although all conditions of the MORPHEUS screen should be inherently cryoprotected.

InlB $_{392}$ T332E, an InlB 392 variant with a single Thr-to-Glu substitution in the B repeat, readily yielded single crystals of varying morphologies in several conditions from the 
commercial MORPHEUS screen and a homemade PEG smear screen (Chaikuad et al., 2015). These crystals reached a size and quality sufficient for data collection without further optimization. The best crystal from condition G2 of the MORPHEUS screen (crystal form I) was a rhombic plate with a cross-section of about $125 \times 50 \mu \mathrm{m}$ and diffracted to $2.05 \AA$ resolution (Table 1). The best crystal from condition D3 of the PEG smear (low- and broad-molecular-weight) screen (crystal form II) was a rod with a cross section of about $35 \times 35 \mu \mathrm{m}$ and a length of about $180 \mu \mathrm{m}$. This crystal diffracted to $1.8 \AA$ resolution (Table 1). We also collected data from InlB $_{392 \_}$T332E crystals grown under different conditions from the MORPHEUS or PEG smear screens. Some were isomorphous to crystal form I and diffracted to lower resolution, so we did not pursue structure determination. Others had larger unit cells and showed signs of translational noncrystallographic symmetry in the native Patterson map. Our attempts to solve these structures by molecular replacement in Phaser both with and without the tNCS option have so far failed.

All three structures described in this paper were easily solved by molecular replacement. Phaser was able to place both the internalin domain and the $\mathrm{B}$ repeat for all five crystallographically independent molecules. The refinement statistics are listed in Table 1. The presence of many weak reflections due to translational pseudosymmetry (see below) may explain the relatively high $R_{\text {work }}$ and $R_{\text {free }}$ values for $\mathrm{InlB}_{392 \_} \mathrm{wt}$.

\subsection{Overall structure}

As expected, there are no major differences between the internalin domains in the $\mathrm{InlB}_{392}$ structures and the previously reported structures of the InlB internalin domain. An overlay of the LRR region revealed some flexibility in the cap and especially in the IR region (Fig. 1b). Likewise, there are no major differences between the B repeats in the $\mathrm{InlB}_{392}$ structures and the published high-resolution structure of the isolated B repeat, with the exception of chain $C$ in the

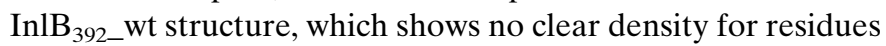
354-372 corresponding to strand $\beta 3$ and the following loop in the other B-repeat structures (Fig. 1c). These residues may be flexible or adopt multiple conformations which cannot be resolved at this rather low resolution of $3.3 \AA$.

The orientation of the $\mathrm{B}$ repeat relative to the internalin domain varies substantially (Figs. $2 a-2 c$ ). There are no polar contacts between the internalin domain and the B repeat to stabilize their arrangement. Many internalins have at least one domain C-terminal to the LRR or IR region (Bierne et al., 2007). Besides InlB, InlK is the only internalin for which a structure extending beyond the internalin domain is known (Neves et al., 2013). The LRR-adjacent D2 domain and the following D3 domain of InlK are structurally distinct from the InlB IR region and B repeat, respectively. Nevertheless, as in our $\mathrm{InlB}_{392}$ structures, few contacts and large flexibility between D2 and D3 were found in InlK (Neves et al., 2013). In InlB, the short linker between the internalin domain and the $\mathrm{B}$ repeat acts as a pivot point rather than a hinge (Figs. $2 a-2 c$ ).

Glu321 was previously regarded as part of the internalin domain (Schubert et al., 2001), but may rather belong to the B repeat, as it forms two polar contacts with other B-repeat residues but none with the internalin domain. In all five crystallographically independent $\operatorname{InlB}_{392}$ chains reported here there are hydrogen bonds between the backbone carbonyl $\mathrm{O}$ atom of Glu321 and the backbone NH of Ala340 and between the side-chain carboxylate of Glu321 and the phenolic $\mathrm{OH}$ of

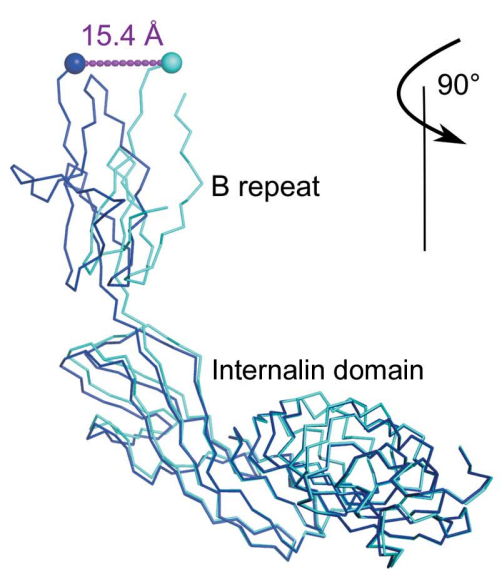

(a)

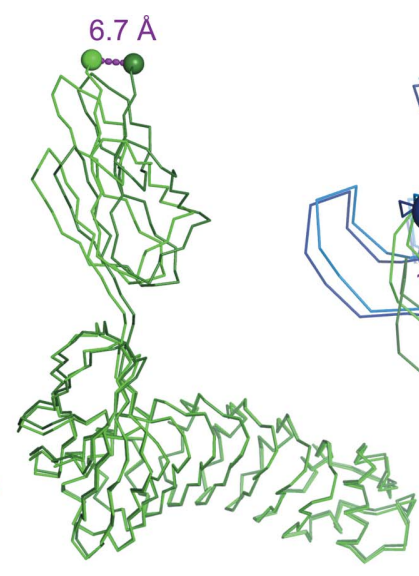

(b)

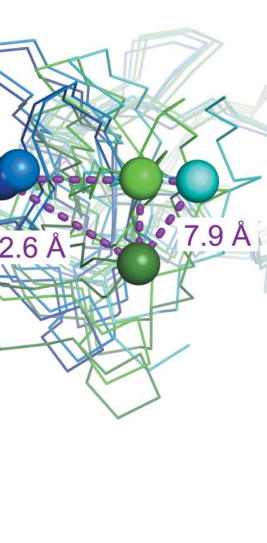

(c)

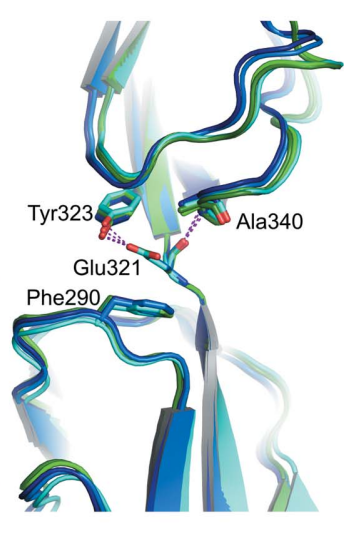

(d)

Figure 2

Movement of the $\mathrm{B}$ repeat relative to the internalin domain. Colouring is as in Fig. 1. Chains $A, B$ and $C$ of InlB ${ }_{392}$ wt are shown in dark blue, blue and cyan, respectively. Crystal forms I and II of $\mathrm{InlB}_{392}$ T332E are shown in green and dark green, respectively. The $\mathrm{C}^{\alpha}$ atoms of the $\mathrm{C}$-terminal residues 391 are shown as spheres. Dashed purple lines indicate the distances between C-terminal residues. (a) Chains $A$ (dark blue) and $C$ (cyan) of InlB 392 wt were aligned on the LRR region. (b) The view is rotated relative to $(a)$ by $90^{\circ}$ around a vertical axis. Crystal forms I (green) and II (dark green) of $\mathrm{InlB}_{392}$ T332E were aligned on the LRR region. (c) Overlay of all InlB 392 structures aligned on the LRR region and shown from the top of the B repeat. (d) Residue Glu321 appears to be an integral part of the B repeat (top) rather than the internalin domain (bottom). Glu321 forms hydrogen bonds from its side chain to the hydroxyl group of Tyr323 and from its backbone carbonyl to the backbone $\mathrm{N}$ atom of Ala340. The only interaction with the internalin domain is a $\mathrm{CH}-\pi$ interaction with Phe290. 
Tyr323 (Fig. 2d). The only interaction of Glu321 and the internalin domain is a $\mathrm{CH}-\pi$ interaction of the aliphatic part of the glutamate side chain with the aromatic ring of Phe290.

\subsection{Crystal packing}

None of the structures contained a twofold-symmetric arrangement of $\mathrm{InlB}_{392}$ indicative of a dimer, and the PISA server (Krissinel \& Henrick, 2007) predicted $\mathrm{InlB}_{392}$ to be monomeric in solution for all three structures. The packing of $\mathrm{InlB}_{392}$ T332E crystal form I is closely related to that of $\mathrm{InlB}_{392}$ wt and the surroundings of all three molecules in the wild-type structure are similar. In a way, the packing of

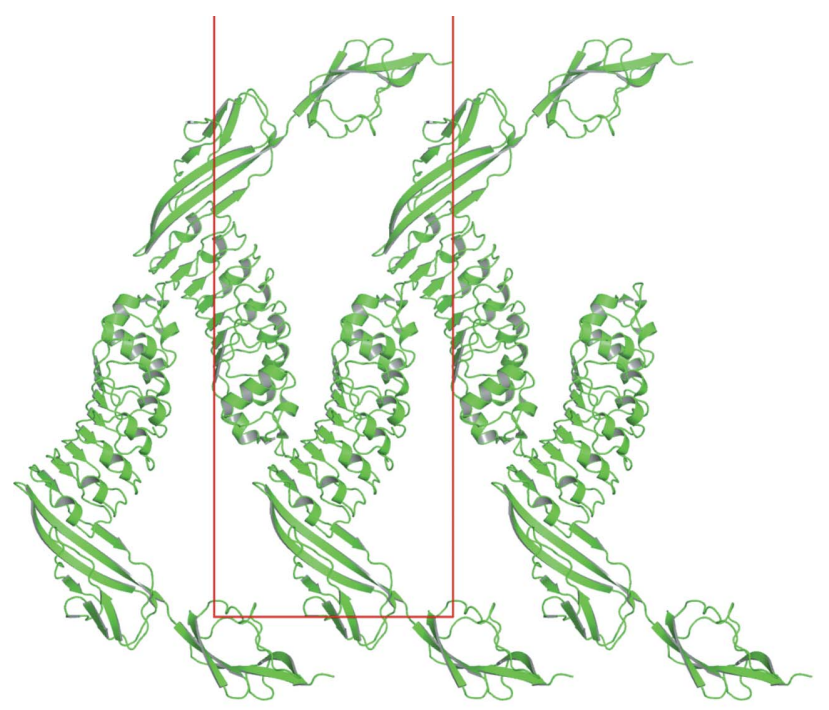

(a)

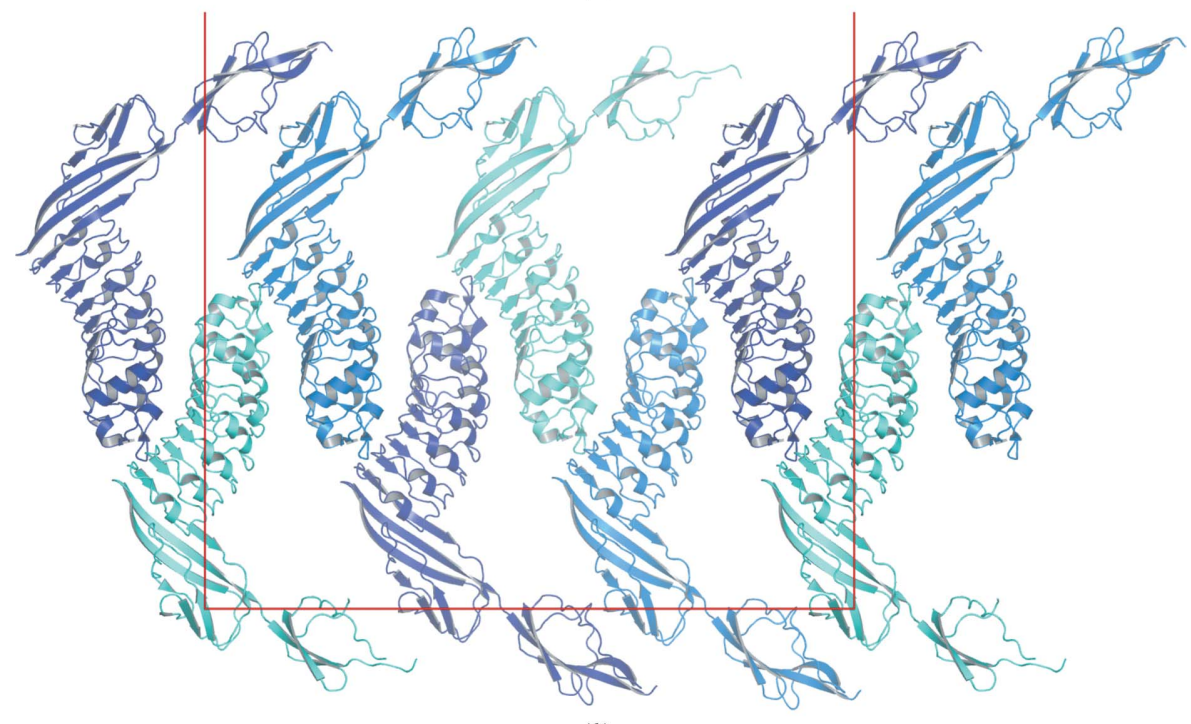

(b)

Figure 3

Crystal packing. ( $a$ ) View along the $a$ axis of InlB $_{392}$ T332E crystal form I. The $b$ axis and part of the $c$ axis (54.59 and $226.72 \AA$ ) are shown horizontally and vertically, respectively. There is one molecule per asymmetric unit. (b) View along the $a$ axis of $\operatorname{InlB}_{392}$ wt. The $b$ axis and part of the $c$ axis $(148.41$ and $220.02 \AA$ ) are shown horizontally and vertically, respectively. There are three molecules per asymmetric unit related by translational noncrystallographic symmetry. The translational component along $b$ is close to $1 / 3$. Chains $A, B$ and $C$ are shown in dark blue, blue and cyan, respectively.
$\mathrm{InlB}_{392 \_ \text {wt }}$ can be viewed as a slightly distorted version of InlB $_{392}$ T332E crystal form I with a tripled $b$ axis (Fig. 3). Accordingly, the native Patterson map of $\mathrm{InlB}_{392}$ wt has an off-origin peak with a peak height of $41.7 \%$ of the origin peak at coordinates $u, v, w=0.0000,0.3234,0.0000$ according to the tNCS detection of Phaser $(38.0 \%$ at $0.000,0.322,0.000$ according to phenix.xtriage). The packing of $\mathrm{InlB}_{392} \mathrm{~T} 332 \mathrm{E}$ crystal form II is different.

A common contact of all four molecules of $\mathrm{InlB}_{392}$ wt and $\mathrm{InlB}_{392}$ T332E crystal form $\mathrm{I}$ is formed between the concave LRR side of one molecule and the cap region and the convex LRR side of another molecule (Fig. 3). The concave side of the LRR is the primary binding site for the MET receptor (Niemann et al., 2007). The crystal contact involves exposed aromatic side chains of InlB that are essential for MET binding (Machner et al., 2003). The contact area is between 397 and $647 \AA^{2}$, with a mean of $549 \AA^{2}$, according to the PISA server (Krissinel $\&$ Henrick, 2007). This crystal contact is not present in crystal form II of

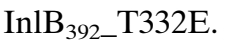

However, all five crystallographically independent $\mathrm{InlB}_{392}$ molecules in the three crystal forms have one recurring crystal contact in common. The contact area lies between 594 and $687 \AA^{2}$, with a mean of $621 \AA^{2}$, according to the PISA server (Krissinel \& Henrick, 2007). This contact is formed between the $\mathrm{B}$ repeat of one molecule and the IR region of a neighbouring molecule (Fig. 4). On the $B$ repeat it involves residues from strand $\beta 2$ (mainly Val329, Thr/Glu332, Val333 and Ile334) and the long loop (residues 347-353) connecting strands $\beta 2$ and $\beta 3$ that is a helix in canonical $\beta$ grasp fold proteins. The PISA server calculates a favourable interaction for this contact (negative solvation-energy effect $\Delta^{\mathrm{i}} G$ ranging from -10.0 to $-23.4 \mathrm{~kJ} \mathrm{~mol}^{-1}$; mean $-15.1 \mathrm{~kJ} \mathrm{~mol}^{-1}$ ), while the previously described LRRcap contact has a positive $\Delta^{\mathrm{i}} G$ ranging from -0.4 to $16.7 \mathrm{~kJ} \mathrm{~mol}^{-1}$ (mean $5.4 \mathrm{~kJ} \mathrm{~mol}^{-1}$ ). The overall arrangement of the contact between the $\mathrm{B}$ repeat and the IR region is similar for all five instances of $\mathrm{InlB}_{392}$ described in this paper. The precise geometry varies somewhat and two groups can be distinguished. One group has three members, namely both crystal forms of $\mathrm{InlB}_{392}$ T332E and the $\mathrm{B}$ repeat of chain $C$ packing against the IR region of chain $A$ in $\mathrm{InlB}_{392}$ wt (Fig. $4 a$ ). The 
second group comprises two crystal contacts of $\mathrm{InlB}_{392 \_w t}$, namely the $\mathrm{B}$ repeat of chain $B$ packing against the IR region of a symmetry-related chain $B$ and the B repeat of chain $A$ packing against the IR region of chain $C$ (Fig. $4 b$ ). The different geometry of the two contact groups is illustrated in Fig. 4(c). In the T332E variant, the mutated residue Glu332 substantially contributes to formation of this crystal contact and is well defined in the electron density (Fig. $4 d$ ).

Taking into account the packing of all three crystal forms described in this paper, the binding of the IR region to the groove between B-repeat strand $\beta 2$ and the loop connecting $\beta 2$ and $\beta 3$ is clearly the dominant packing interaction. This crystal contact has the largest mean interface area, the most negative $\Delta^{\mathrm{i}} G$ and is formed by all molecules. Potential functional implications will be considered in Section 4.

\section{Discussion}

4.1. The T332E mutation has no impact on the B-repeat structure

The T332E substitution is one of two point mutations that we had previously found to have a negative effect on the biological function of the $\mathrm{B}$ repeat (Bleymüller et al., 2016). Thr332 is surface-exposed. Therefore, we had expected the mutation to glutamate not to impair the B-repeat structure. The circular-dichroism spectrum and the elution behaviour on a gel-filtration column confirmed this assumption, as they were basically identical for the wild-type B repeat and the T332E variant (Bleymüller et al., 2016). The crystal structures presented in this work provide additional and conclusive proof that the T332E mutation does not change the structure of the $\mathrm{B}$ repeat. Therefore, the negative effect of this substitution in cellular assays is most likely due to the destruction of a binding site, preventing the interaction with a functionally important binding partner.

\subsection{Effect of the T332E mutation on crystallization}

Intriguingly, wild-type $\mathrm{InlB}_{392}$ was a much more problematic crystallization target than the $\mathrm{InlB}_{392} \mathrm{~T} 332 \mathrm{E}$ variant. This is unexpected because glutamate side chains are statistically underrepresented in interfaces of oligomeric proteins, presumably due to their high conformational entropy (Derewenda \& Vekilov, 2006). Moreover, crystal contacts are systematically depleted of residues with high side-chain entropy, and Glu, along with Lys, has the lowest propensity to form crystal contacts 
(Cieślik \& Derewenda, 2009). In the semi-rational surfaceentropy reduction (SER) approach, surface-exposed glutamates are mutated to alanine, threonine or tyrosine in order to

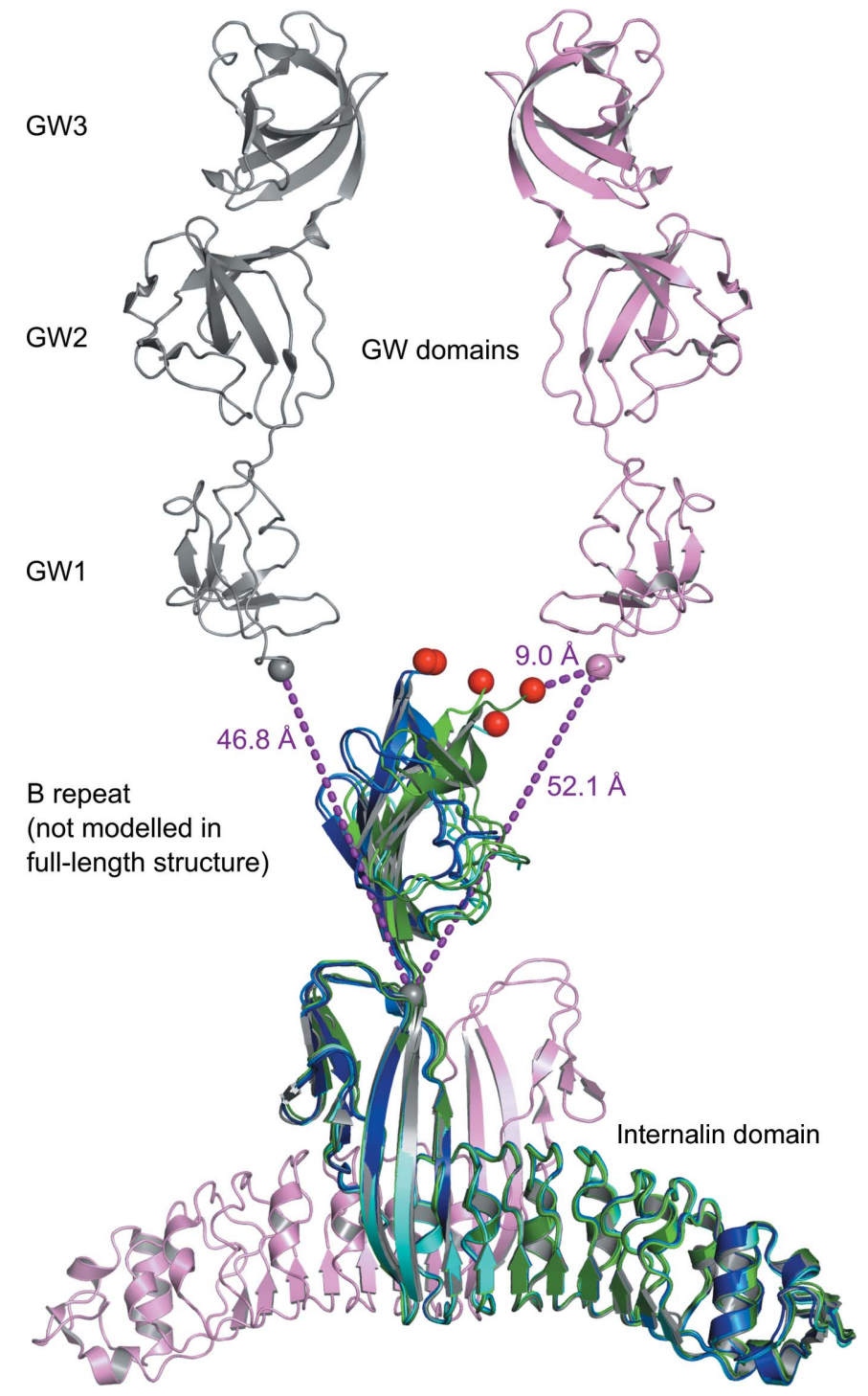

Figure 5

Comparison of $\mathrm{InlB}_{392}$ structures with the structure of full-length InlB (PDB entry 1m9s). Two symmetry-related copies of full-length InlB are shown in grey and pink, with the internalin domains at the bottom and the three GW domains at the top. $\mathrm{C}^{\alpha}$ atoms of the last residue of the grey internalin domain (residue 319) and of residue 393 at the beginning of the GW domains are shown as spheres. Because there was no interpretable density for the B repeat, it was unclear which GW domains should be grouped with the grey internalin domain. Ghosh and coworkers deposited the asymmetric unit containing a combination of the internalin domain and GW domains with the shorter distance. The other choice of asymmetric unit (grey internalin domain and pink GW domains) would result in only a slightly longer distance. All InlB $_{392}$ structures were structurally aligned on the grey internalin domain. Colouring is as in Fig. 1. Chains $A, B$ and $C$ of $\mathrm{InlB}_{392}$ wt are shown in dark blue, blue and cyan, respectively. Crystal forms I and II of $\mathrm{InIB}_{392}$ T332E are shown in green and dark green, respectively. $\mathrm{C}^{\alpha}$ atoms of the $\mathrm{C}$-terminal residues (391 or 392) are shown as red spheres. Crystal form II of InlB 392 T332E has the shortest distance of $9.0 \mathrm{~A}$ between its C-terminal residue 392 and residue 393 of the pink GW domains. All distances are shown as purple dashed lines. increase the likelihood of crystallization (Mateja et al., 2002; Cooper et al., 2007). Analysis of the actual crystal contacts with the PISA server indicates an unfavourable contribution of Thr332 (positive solvation-energy effect $\Delta^{\mathrm{i}} G \simeq$ $\left.4.2 \mathrm{~kJ} \mathrm{~mol}^{-1}\right)$ but an almost neutral effect of Glu332 $\left(\Delta^{\mathrm{i}} G \simeq\right.$ $\left.0 \mathrm{~kJ} \mathrm{~mol}^{-1}\right)$. One explanation for this unexpected effect of the T332E substitution would be that the B repeat evolved to prevent fortuitous binding to the IR domain and that the T332E mutation counteracts this anti-aggregation property.

\subsection{Comparison with the structure of full-length InIB}

In the structure of full-length InlB there were two possibilities to connect the GW domains to the internalin domain, with similar distances between the C-terminal residue of the internalin domain and the $\mathrm{N}$-terminal residue of the $\mathrm{GW}$ domains (Marino et al., 2002). The authors deposited an asymmetric unit (shown in grey in Fig. 5) containing the combination with the slightly shorter distance of $47 \AA$ between the $\mathrm{C}^{\alpha}$ atoms of residues 319 and 393 in the Protein Data Bank. The distance to residue 393 of a symmetry-related copy of the GW domains (shown in pink in Fig. 5) is $52 \AA$.

Upon overlaying all five copies of $\mathrm{InlB}_{392}$ described in this work with one copy of the InlB full-length dimer, the C-terminal residues of the $\mathrm{B}$ repeat are about halfway between the two possible GW domains (Fig. 5). For chain $A$ of $\mathrm{InlB}_{392}$ wt, the distances to residue 393 of the GW domains of the deposited asymmetric unit and the symmetry-related GW domains are 22.1 and $20.8 \AA$, respectively. The largest difference between these distances is found for $\mathrm{InlB}_{392} \mathrm{~T} 332 \mathrm{E}$ crystal form II. The distances to residue 393 of the GW domains of the deposited asymmetric unit and the symmetryrelated GW domains of PDB entry $1 \mathrm{~m} 9 \mathrm{~s}$ (Marino et al., 2002) are 34.4 and $9.0 \AA$, respectively. For all five instances of $\operatorname{InlB}_{392}$ reported here, the shortest distance between the C-terminal residue of the $\mathrm{B}$ repeat and the $\mathrm{N}$-terminal residue of the $\mathrm{GW}$ domains is found for the symmetry-related GW domains. However, none of the orientations of the B repeat observed in $\mathrm{InlB}_{392}$ can represent the position present in the full-length protein in PDB entry $1 \mathrm{~m} 9 \mathrm{~s}$, as no residues are left that could span the distance of at least $9.0 \AA$. Moreover, if one overlays two copies of $\mathrm{InlB}_{392}$ onto the two copies of the functional fulllength dimer, there would be severe clashes of the B repeat (Supplementary Fig. S1). Given the highly flexible linkage between the internalin domain and the $\mathrm{B}$ repeat, this suggests that crystal-packing forces govern the observed orientations of the $\mathrm{B}$ repeat.

\subsection{Strand $\beta 2$ of the B repeat may be a 'sticky patch' favouring cohesive interactions}

Strikingly, the contact between B-repeat strand $\beta 2$ and the IR region of a neighbouring molecule is similar in all five chains. This is unusual as monomeric proteins rarely have common interfaces in more than one-third of their crystal forms and generally much less (Xu et al., 2008). Recurring crystal contacts indicate energetically favourable interactions and sometimes they are even biologically relevant (Xu et al., 


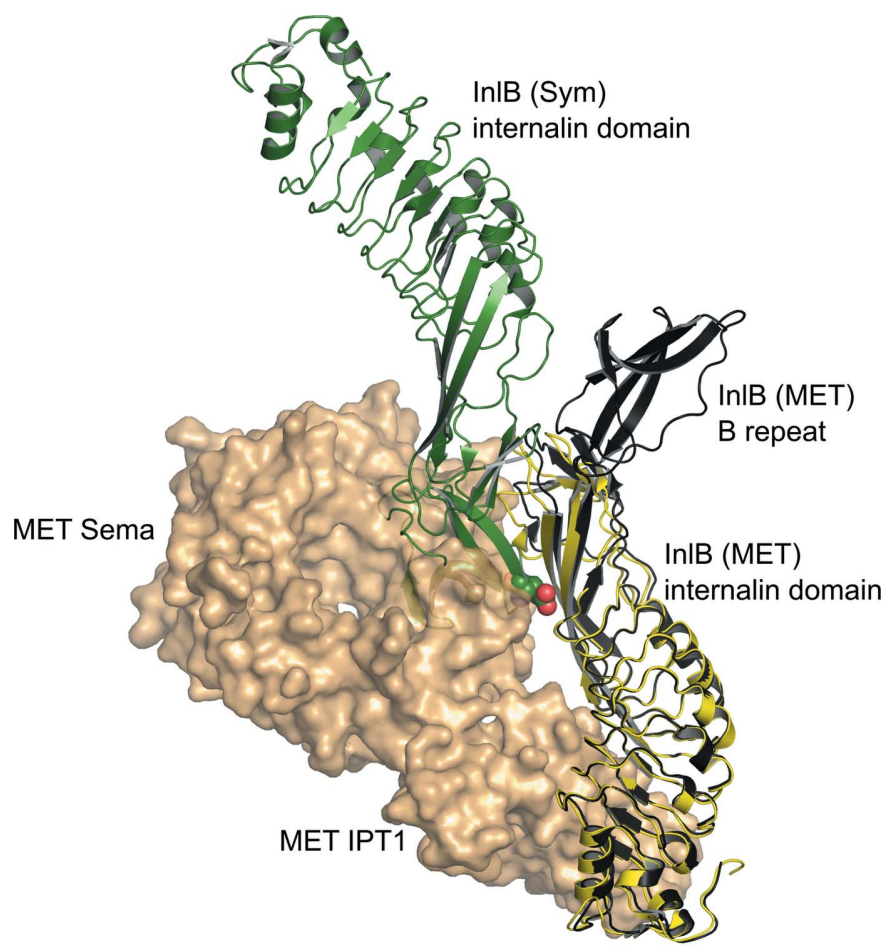

(a)

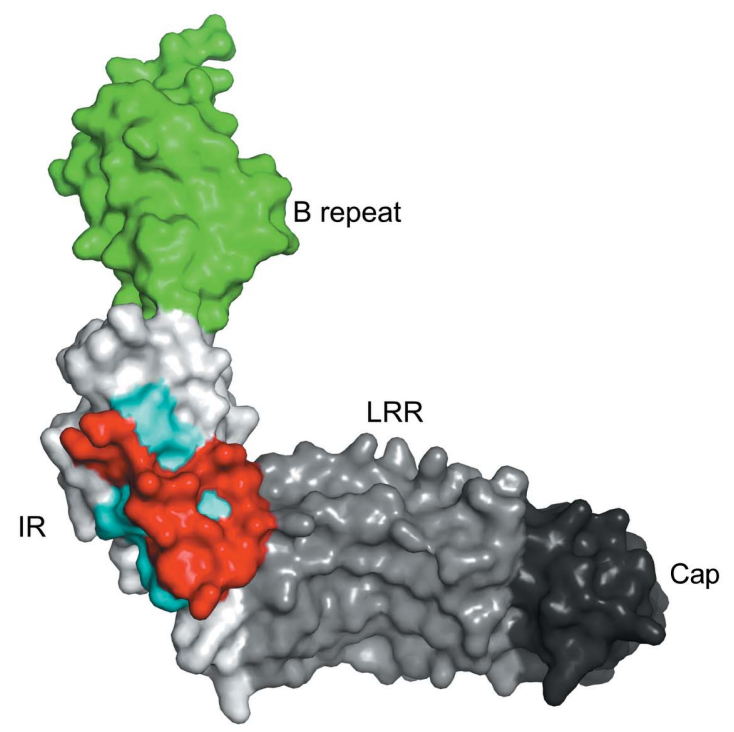

(b)

Figure 6

The same residues of the IR region bind the MET Sema domain in the InlB-MET complex and form the crystal contact to the B repeat in InlB $_{392}$. (a) Overlay of the InlB-MET complex (PDB entry 2uzx) and $\mathrm{InlB}_{392}$ (PDB entry $7 \mathrm{nms}$ ). MET is shown as an orange surface. $\mathrm{InlB}_{392}$ (grey) was structurally aligned with the InlB internalin domain (yellow) from the InlB-MET complex. A symmetry-related InlB $_{392}$ is shown in dark green. The B repeat of this symmetry-related $\mathrm{InlB}_{392}$ overlaps with the MET Sema domain where it contacts the IR region. Glu332 that is mutated in the T332E variant is shown as spheres. (b) $\mathrm{InlB}_{392}$ (PDB entry $7 \mathrm{nms}$ ) is shown as a surface. The cap region is coloured black, the LRR region grey, the IR region white and the $\mathrm{B}$ repeat green. Residues of the IR region involved in both binding of the MET Sema domain and formation of the crystal contact with the $\mathrm{B}$ repeat are shown in red. Residues only involved in the crystal contact with the B repeat are coloured cyan.
2008). The contact between strand $\beta 2$ and the IR region most likely does not represent a physiological contact. Firstly, in the complex with the MET receptor (Niemann et al., 2007) the IR region contacts the Sema domain of the MET receptor with residues that contact strand $\beta 2$ of the $\mathrm{B}$ repeat in the $\mathrm{InlB}_{392}$ structures (Fig. 6). Upon binding to MET, the IR region will therefore not be able to form this contact with the B repeat. Secondly, the contact between the B repeat and the IR region is formed regardless of the T332E mutation. As the T332E mutation strongly impairs the biological function of the $\mathrm{B}$ repeat in cellular assays, it appears highly unlikely that this represents a physiologically relevant contact.

Instead, we assume that this is a fortuitous interaction between two binding sites lacking their native binding partner. The MET binding site in the IR region and strand $\beta 2$ of the $\mathrm{B}$ repeat may thus represent 'sticky patches', i.e. specific surface patches with properties that are thermodynamically favourable for cohesive interactions (Derewenda \& Godzik, 2017). This hypothesis is supported by the $\Delta^{\mathrm{i}} G P$-value reported by the $P I S A$ server. The $\Delta^{\mathrm{i}} G P$-value is a measure of interface specificity. A $P$-value larger than 0.5 means that the interface is less hydrophobic than it could be and therefore the interface is likely to be an artefact of crystal packing. A $P$-value smaller than 0.5 indicates an interface with a hydrophobicity that is

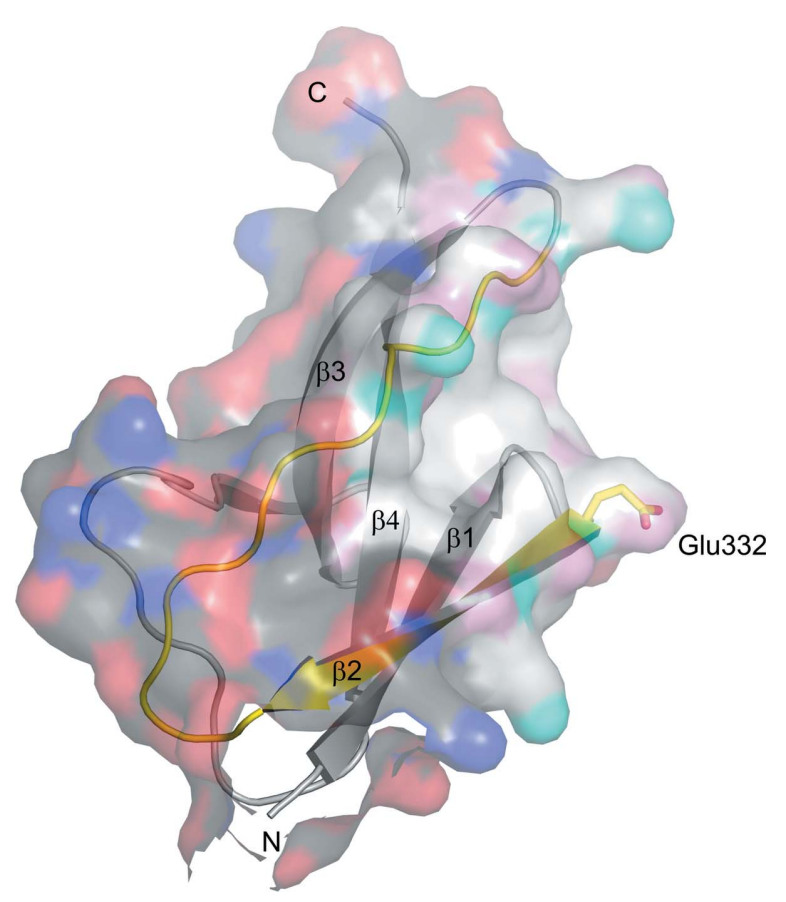

Figure 7

B-repeat residues forming the crystal contact to the IR region may belong to a 'sticky patch' in the hydrophobic groove between strand $\beta 2$ and the following loop (both coloured yellow in the cartoon). The $\mathrm{B}$ repeat of $\mathrm{InlB}_{392}$ T332E (PDB entry $7 \mathrm{nms}$ ) is shown in cartoon representation with a transparent surface. The surface is coloured according to element, with $\mathrm{C}$ atoms in dark grey, $\mathrm{N}$ atoms in blue and $\mathrm{O}$ atoms in red. Residues involved in formation of the crystal contact with the IR region in InlB $_{392}$ T332E (PDB entry $7 \mathrm{nms}$ ) are shown in lighter colours with $\mathrm{C}$ atoms in white, $\mathrm{N}$ atoms in cyan and $\mathrm{O}$ atoms in pink. The white patch highlights the apolar nature of the groove between strand $\beta 2$ and the following loop. 
higher than the average for the given structures would be, implying that the interface surface can be interaction-specific (Krissinel \& Henrick, 2007). The cap-LRR interface that is present in crystal form I of the T332E variant and three times in the wild-type structure has a mean $\Delta^{\mathrm{i}} G P$-value of 0.62 , indicating a pure crystal-packing contact. In contrast, the interface between the $\mathrm{B}$ repeat and the IR region that is present in all structures has a $\Delta^{\mathrm{i}} G P$-value of 0.28 , indicating higher hydrophobicity than would be expected on average (Fig. 7).

We previously observed a similar phenomenon for the $Y$. enterocolitica type III secretion protein SycD. Recombinantly produced SycD showed weak, concentration-dependent homodimerization in solution. Two crystal forms showed putative homodimers; however, the arrangement of protomers in these dimers differed (Büttner et al., 2008). Both homodimers involved similar surface patches in the first tetratricopeptide repeat (TPR), but the geometry of the two contacts differed. Subsequently, a structure of the complex of the Aeromonas hydrophila homolog $\mathrm{AscH}$ with AopB suggested that SycD probably employs this region of TPR1 for interaction with its binding partner YopB, when present (Nguyen et al., 2015). In the absence of YopB, the YopB binding site in TPR1 of SycD could be a 'sticky patch' that promotes fortuitous $\mathrm{SycD}$ homodimerization and supports different geometries of the dimer interface.

Our assumption that the hydrophobic groove formed by strand $\beta 2$ and the loop connecting strands $\beta 2$ and $\beta 3$ forms the primary ligand-binding site in the B repeat (Fig. 7) is supported by our previous observations. In an attempt to identify potential binding sites in the $\mathrm{B}$ repeat by mutagenesis, the only two substitutions of surface residues that resulted in a loss of function were located in strand $\beta 2$. We had suggested that the $\mathrm{B}$ repeat potentiates MET activation by forming a weak homodimer contact through which it could promote the dimerization of MET bound to the internalin domain. The T332E mutation would prevent homodimerization of the B repeat and thereby suppress MET dimerization through the internalin domain (Bleymüller et al., 2016). The $\operatorname{InlB}_{392}$ structures presented here are compatible with the role of the B repeat in MET activation that we proposed previously. However, additional experiments will be required to corroborate or disprove this model, as we did not observe homodimerization of the $\mathrm{B}$ repeat even in crystals of wild-type $\mathrm{InlB}_{392}$.

\section{Conclusion: crystal contacts as valuable assets}

While crystal-packing contacts are often suspected to lead to structural artefacts, they can also help to reveal functional information when comparing different packing environments. Here, five crystallographically independent molecules reveal protein dynamics, highlighting the high inter-domain flexibility between the internalin domain and the $\mathrm{B}$ repeat of InlB. However, the five structures presented here apparently do not completely map out the conformational space of $\mathrm{InlB}_{392}$, as the $\mathrm{B}$ repeat needs to adopt yet another position to fit into the structure of full-length InlB. Therefore, additional crystal forms and further structures are likely to show even larger inter-domain movements.

The largest packing contact that is similarly formed by all five crystallographically independent instances of $\mathrm{InlB}_{392}$ involves the known binding site for the MET Sema domain in the IR region and a surface patch in the B repeat that has previously been shown to be functionally important through mutagenesis and cellular assays. The structures analysed in this paper thus represent a good example showing that recurrent crystal contacts can highlight physiologically relevant binding sites. Due to the extremely high protein concentration in crystals, crystallography allows the visualization of protein-protein interactions that are too weak to be studied in solution. The biological relevance of such contacts needs to be addressed by complementary functional assays (Kobe et al., 2008). If an actual binding partner is missing, the interface area can still show up as a crystal contact interacting with heterologous protein surfaces in a nonphysiological way (Forwood et al., 2007). In a time where protein crystallography is increasingly sandwiched between accurate protein structure prediction (Baek et al., 2021; Jumper et al., 2021) and highresolution cryo-EM of large proteins and complexes (Kühlbrandt, 2014), the careful analysis of crystal contacts might turn out to be a valuable asset for crystallography.

\section{Acknowledgements}

Synchrotron MX data were collected on beamline P13 operated by EMBL Hamburg at the PETRA III storage ring, DESY, Hamburg, Germany. We would like to thank Dr Isabel Bento and Saravanan Panneerselvam for assistance in using the beamline. Measurements were carried out on the BL14.2 beamline at the BESSY II electron-storage ring operated by the Helmholtz-Zentrum Berlin für Materialien und Energie. We would like to thank Dr Christian Feiler for assistance during the experiment. Open access funding enabled and organized by Projekt DEAL.

\section{Funding information}

We gratefully acknowledge the financial support from HZB.

\section{References}

Andres, F., Iamele, L., Meyer, T., Stüber, J. C., Kast, F., Gherardi, E., Niemann, H. H. \& Plückthun, A. (2019). J. Mol. Biol. 431, 20202039.

Baek, M., DiMaio, F., Anishchenko, I., Dauparas, J., Ovchinnikov, S., Lee, G. R., Wang, J., Cong, Q., Kinch, L. N., Schaeffer, R. D., Millán, C., Park, H., Adams, C., Glassman, C. R., DeGiovanni, A., Pereira, J. H., Rodrigues, A. V., van Dijk, A. A., Ebrecht, A. C., Opperman, D. J., Sagmeister, T., Buhlheller, C., Pavkov-Keller, T., Rathinaswamy, M. K., Dalwadi, U., Yip, C. K., Burke, J. E., Garcia, K. C., Grishin, N. V., Adams, P. D., Read, R. J. \& Baker, D. (2021). Science, 373, 871-876.

Banerjee, M., Copp, J., Vuga, D., Marino, M., Chapman, T., Van Der Geer, P. \& Ghosh, P. (2004). Mol. Microbiol. 52, 257-271.

Bierne, H., Sabet, C., Personnic, N. \& Cossart, P. (2007). Microbes Infect. 9, 1156-1166. 
Birchmeier, C., Birchmeier, W., Gherardi, E. \& Vande Woude, G. F. (2003). Nat. Rev. Mol. Cell Biol. 4, 915-925.

Bleymüller, W. M., Lämmermann, N., Ebbes, M., Maynard, D., Geerds, C. \& Niemann, H. H. (2016). J. Biol. Chem. 291, 2556725577.

Büttner, C. R., Sorg, I., Cornelis, G. R., Heinz, D. W. \& Niemann, H. H. (2008). J. Mol. Biol. 375, 997-1012.

Casañal, A., Lohkamp, B. \& Emsley, P. (2020). Protein Sci. 29, 10691078.

Chaikuad, A., Knapp, S. \& von Delft, F. (2015). Acta Cryst. D71, 1627-1639.

Cianci, M., Bourenkov, G., Pompidor, G., Karpics, I., Kallio, J., Bento, I., Roessle, M., Cipriani, F., Fiedler, S. \& Schneider, T. R. (2017). J. Synchrotron Rad. 24, 323-332.

Cieślik, M. \& Derewenda, Z. S. (2009). Acta Cryst. D65, 500-509.

Cooper, D. R., Boczek, T., Grelewska, K., Pinkowska, M., Sikorska, M., Zawadzki, M. \& Derewenda, Z. (2007). Acta Cryst. D63, 636645.

Copp, J., Marino, M., Banerjee, M., Ghosh, P. \& van der Geer, P. (2003). J. Biol. Chem. 278, 7783-7789.

Derewenda, Z. S. \& Godzik, A. (2017). Methods Mol. Biol. 1607, 77115.

Derewenda, Z. S. \& Vekilov, P. G. (2006). Acta Cryst. D62, 116-124.

Diederichs, K., McSweeney, S. \& Ravelli, R. B. G. (2003). Acta Cryst. D59, 903-909.

Dietz, M. S., Hasse, D., Ferraris, D. M., Göhler, A., Niemann, H. H. \& Heilemann, M. (2013). BMC Biophys. 6, 6.

Dramsi, S., Biswas, I., Maguin, E., Braun, L., Mastroeni, P. \& Cossart, P. (1995). Mol. Microbiol. 16, 251-261.

Ebbes, M., Bleymüller, W. M., Cernescu, M., Nölker, R., Brutschy, B. \& Niemann, H. H. (2011). J. Biol. Chem. 286, 15496-15506.

Ferraris, D. M., Gherardi, E., Di, Y., Heinz, D. W. \& Niemann, H. H. (2010). J. Mol. Biol. 395, 522-532.

Forwood, J. K., Thakur, A. S., Guncar, G., Marfori, M., Mouradov, D., Meng, W., Robinson, J., Huber, T., Kellie, S., Martin, J. L., Hume, D. A. \& Kobe, B. (2007). Proc. Natl Acad. Sci. USA, 104, 1038210387.

Gorrec, F. (2009). J. Appl. Cryst. 42, 1035-1042.

Harwardt, M.-L. I. E., Young, P., Bleymüller, W. M., Meyer, T., Karathanasis, C., Niemann, H. H., Heilemann, M. \& Dietz, M. S. (2017). FEBS Open Bio, 7, 1422-1440.

Jonquières, R., Bierne, H., Fiedler, F., Gounon, P. \& Cossart, P. (1999). Mol. Microbiol. 34, 902-914.

Jonquières, R., Pizarro-Cerdá, J. \& Cossart, P. (2001). Mol. Microbiol. 42, 955-965.

Jumper, J., Evans, R., Pritzel, A., Green, T., Figurnov, M., Ronneberger, O., Tunyasuvunakool, K., Bates, R., Žídek, A., Potapenko, A., Bridgland, A., Meyer, C., Kohl, S. A. A., Ballard, A. J., Cowie, A., Romera-Paredes, B., Nikolov, S., Jain, R., Adler, J., Back, T., Petersen, S., Reiman, D., Clancy, E., Zielinski, M., Steinegger, M., Pacholska, M., Berghammer, T., Bodenstein, S., Silver, D., Vinyals, O., Senior, A. W., Kavukcuoglu, K., Kohli, P. \& Hassabis, D. (2021). Nature, 596, 583-589.

Kabsch, W. (2010). Acta Cryst. D66, 125-132.

Kobe, B., Guncar, G., Buchholz, R., Huber, T., Maco, B., Cowieson, N., Martin, J. L., Marfori, M. \& Forwood, J. K. (2008). Biochem. Soc. Trans. 36, 1438-1441.

Koschut, D., Richert, L., Pace, G., Niemann, H. H., Mély, Y. \& OrianRousseau, V. (2016). Biochim. Biophys. Acta, 1863, 1552-1558.
Kovalevskiy, O., Nicholls, R. A., Long, F., Carlon, A. \& Murshudov, G. N. (2018). Acta Cryst. D74, 215-227.

Krissinel, E. \& Henrick, K. (2007). J. Mol. Biol. 372, 774-797.

Kühlbrandt, W. (2014). Science, 343, 1443-1444.

Liebschner, D., Afonine, P. V., Baker, M. L., Bunkóczi, G., Chen, V. B., Croll, T. I., Hintze, B., Hung, L.-W., Jain, S., McCoy, A. J., Moriarty, N. W., Oeffner, R. D., Poon, B. K., Prisant, M. G., Read, R. J., Richardson, J. S., Richardson, D. C., Sammito, M. D., Sobolev, O. V., Stockwell, D. H., Terwilliger, T. C., Urzhumtsev, A. G., Videau, L. L., Williams, C. J. \& Adams, P. D. (2019). Acta Cryst. D75, 861-877.

Lingnau, A., Domann, E., Hudel, M., Bock, M., Nichterlein, T., Wehland, J. \& Chakraborty, T. (1995). Infect. Immun. 63, 38963903.

Machner, M. P., Frese, S., Schubert, W. D., Orian-Rousseau, V., Gherardi, E., Wehland, J., Niemann, H. H. \& Heinz, D. W. (2003). Mol. Microbiol. 48, 1525-1536.

Marino, M., Banerjee, M., Jonquières, R., Cossart, P. \& Ghosh, P. (2002). EMBO J. 21, 5623-5634.

Marino, M., Braun, L., Cossart, P. \& Ghosh, P. (1999). Mol. Cell, 4, 1063-1072.

Mateja, A., Devedjiev, Y., Krowarsch, D., Longenecker, K., Dauter, Z., Otlewski, J. \& Derewenda, Z. S. (2002). Acta Cryst. D58, 1983 1991.

McCoy, A. J., Grosse-Kunstleve, R. W., Adams, P. D., Winn, M. D., Storoni, L. C. \& Read, R. J. (2007). J. Appl. Cryst. 40, 658-674.

Mueller, U., Förster, R., Hellmig, M., Huschmann, F. U., Kastner, A., Malecki, P., Pühringer, S., Röwer, M., Sparta, K., Steffien, M., Ühlein, M., Wilk, P. \& Weiss, M. S. (2015). Eur. Phys. J. Plus, 130, 141.

Neves, D., Job, V., Dortet, L., Cossart, P. \& Dessen, A. (2013). J. Mol. Biol. 425, 4520-4529.

Nguyen, V. S., Jobichen, C., Tan, K. W., Tan, Y. W., Chan, S. L., Ramesh, K., Yuan, Y., Hong, Y., Seetharaman, J., Leung, K. Y., Sivaraman, J. \& Mok, Y. K. (2015). Structure, 23, 2022-2031.

Niemann, H. H. (2013). Biochim. Biophys. Acta, 1834, 2195-2204.

Niemann, H. H., Jäger, V., Butler, P. J. G., van den Heuvel, J., Schmidt, S., Ferraris, D., Gherardi, E. \& Heinz, D. W. (2007). Cell, 130, 235246.

Pizarro-Cerdá, J., Kühbacher, A. \& Cossart, P. (2012). Cold Spring Harb. Perspect. Med. 2, a010009.

Schubert, W. D., Göbel, G., Diepholz, M., Darji, A., Kloer, D., Hain, T., Chakraborty, T., Wehland, J., Domann, E. \& Heinz, D. W. (2001). J. Mol. Biol. 312, 783-794.

Seveau, S., Tham, T. N., Payrastre, B., Hoppe, A. D., Swanson, J. A. \& Cossart, P. (2007). Cell. Microbiol. 9, 790-803.

Shen, Y., Naujokas, M., Park, M. \& Ireton, K. (2000). Cell, 103, 501510.

Trusolino, L., Bertotti, A. \& Comoglio, P. M. (2010). Nat. Rev. Mol. Cell Biol. 11, 834-848.

Winn, M. D., Ballard, C. C., Cowtan, K. D., Dodson, E. J., Emsley, P., Evans, P. R., Keegan, R. M., Krissinel, E. B., Leslie, A. G. W., McCoy, A., McNicholas, S. J., Murshudov, G. N., Pannu, N. S., Potterton, E. A., Powell, H. R., Read, R. J., Vagin, A. \& Wilson, K. S. (2011). Acta Cryst. D67, 235-242.

Xu, Q., Canutescu, A. A., Wang, G., Shapovalov, M., Obradovic, Z. \& Dunbrack, R. L. (2008). J. Mol. Biol. 381, 487-507. 